\title{
GAMMA-RAY PULSAR STUDIES WITH COMPTEL
}

\author{
W. HERMSEN AND L. KUIPER \\ SRON-Leiden, Postbus 9504, 2300 RA Leiden, The Netherlands \\ R. DieHL, G. LichtI, V. SCHÖNFELDER, AND A. W. STRONG \\ Max-Planck Institut für Extraterrestrische Physik, Postfach 1603, D-85740 Garching, Germany
}

A. CONNORS AND J. RYAN

Space Science Center, University of New Hampshire, Durham, NH 03824

K. BENNETt, M. BusetTA, AND A. CaRramiñana

Astrophysics Division, European Space Research and Technology Centre, 2200 AG Noordwijk, The Netherlands

\section{R. BUCCHERI}

IFCAI/CNR, Piazza G. Verdi 6, 90139 Palermo, Italy

AND

I. A. GRENIER

EUROPA, Université Paris 7, Observatoire de Paris, France; DAPNIA/SAp, Centre d'Etudes de Saclay, France Received 1993 April 12; accepted 1993 August 20

\begin{abstract}
Since the launch of the Compton Gamma-Ray Observatory (CGRO) the number of detected $\gamma$-ray pulsars increased from two to six. COMPTEL, on-board $C G R O$ and sensitive to $\gamma$-rays with energies between $\sim 0.7$ and $30 \mathrm{MeV}$, detected three of these unambiguously. The classical Crab and Vela pulsars have been observed on several occasions and detailed pulse patterns and spectral parameters have been derived. The new $C G R O \gamma$-ray pulsar PSR B1509-58 has been detected by COMPTEL at a significance level above $4 \sigma$, consistently in a timing and spatial analysis. A likely detection of Geminga has been obtained at a $\sim 3 \sigma$ level. This indication is found in a phase interval in which $\operatorname{COS} B$ data showed the presence of a new variable component, Interpeak 2, exhibiting a very soft spectrum above $50 \mathrm{MeV}$. The diversities in light-curve shapes and spectral distributions, the apparent time variabilities, and the significant differences in the fractions of the spin-down power radiated at $\gamma$-ray energies in this small sample of $\gamma$-ray pulsars pose important constraints to pulsar modeling.
\end{abstract}

Subject headings: gamma rays: observations - pulsars: general

\section{INTRODUCTION}

During the all-sky-survey period of the Compton GammaRay Observatory (CGRO), the number of known $\gamma$-ray pulsars has tripled. Prior to the launch of $C G R O$, only two radio pulsars were established $\gamma$-ray pulsars at energies above $\sim 1$ MeV: the Crab pulsar (PSR B0531+21) and the Vela pulsar (PSR B0833-45), exhibiting very different $\gamma$-ray spectra in contrast to the similarity in the shape of their $\gamma$-ray light curves (e.g., Clear et al. 1987, for PSR B0531+21; Grenier, Hermsen, $\&$ Clear 1988, for PSR B0833-45). Four additional $\gamma$-ray pulsars have been found of which three were known radio pulsars, namely: PSR B1509-58 (Wilson et al. 1991), PSR B1706-44 (Thompson et al. 1992), and PSR B1055-52 (Fierro et al. 1993; Grenier et al. 1994c). Finally, the enigmatic source Geminga was discovered to exhibit periodicity at $237 \mathrm{~ms}$ in the ROSAT X-ray data by Halpern \& Holt (1992), later confirmed in the $\gamma$-ray data recorded by EGRET, $\operatorname{COS} B, S A S 2$, and Gamma 1 (Bertsch et al. 1992; Bignami \& Caraveo 1992; Hermsen et al. 1992; Akimov et al. 1993). So far Geminga has not been seen in the radio domain.

The diversity in light-curve and spectral shapes in this small sample of $\gamma$-ray pulsars is remarkable. These new results complicate the picture of the production of pulsed $\gamma$-rays, whether it is based on a polar-cap (Daugherthy \& Harding 1982) or an outer-gap model (Cheng, Ho, \& Ruderman 1986a,b). Therefore, any additional experimental results are valuable for model building. Particularly, the determination of source characteristics such as spectral shapes, breaks and time variability as a function of pulsar phase over the whole of the $C G R O$ energy domain provides important diagnostic tools. The COMPTEL telescope aboard CGRO is sensitive in the middle energy range between about 0.7 and $30 \mathrm{MeV}$. This paper presents the COMPTEL results on pulsar studies. Detailed information has been obtained on the "classical" $\gamma$-ray pulsars Crab (Strong et al. 1993; Bennett et al. 1993) and Vela (Hermsen et al. 1993). These will be summarized. Furthermore, evidence at the 3-4 $\sigma$ level will be presented for low-energy $\gamma$-ray emission from PSR B1509-58 (Bennett et al. 1994) and Geminga. Finally, the general search for pulsars in the COMPTEL data will be discussed.

\section{THE CRAB AND VELA PULSARS}

During the all-sky survey COMPTEL has viewed the Crab and Vela several times. With the benefit of contemporary ephemerides (Johnston et al. 1992), phase-folding has produced light curves for both pulsars as seen in Figure 1. These light curves evidently show different structures, as has been discussed earlier by, e.g., Bennett et al. (1994). For example: 

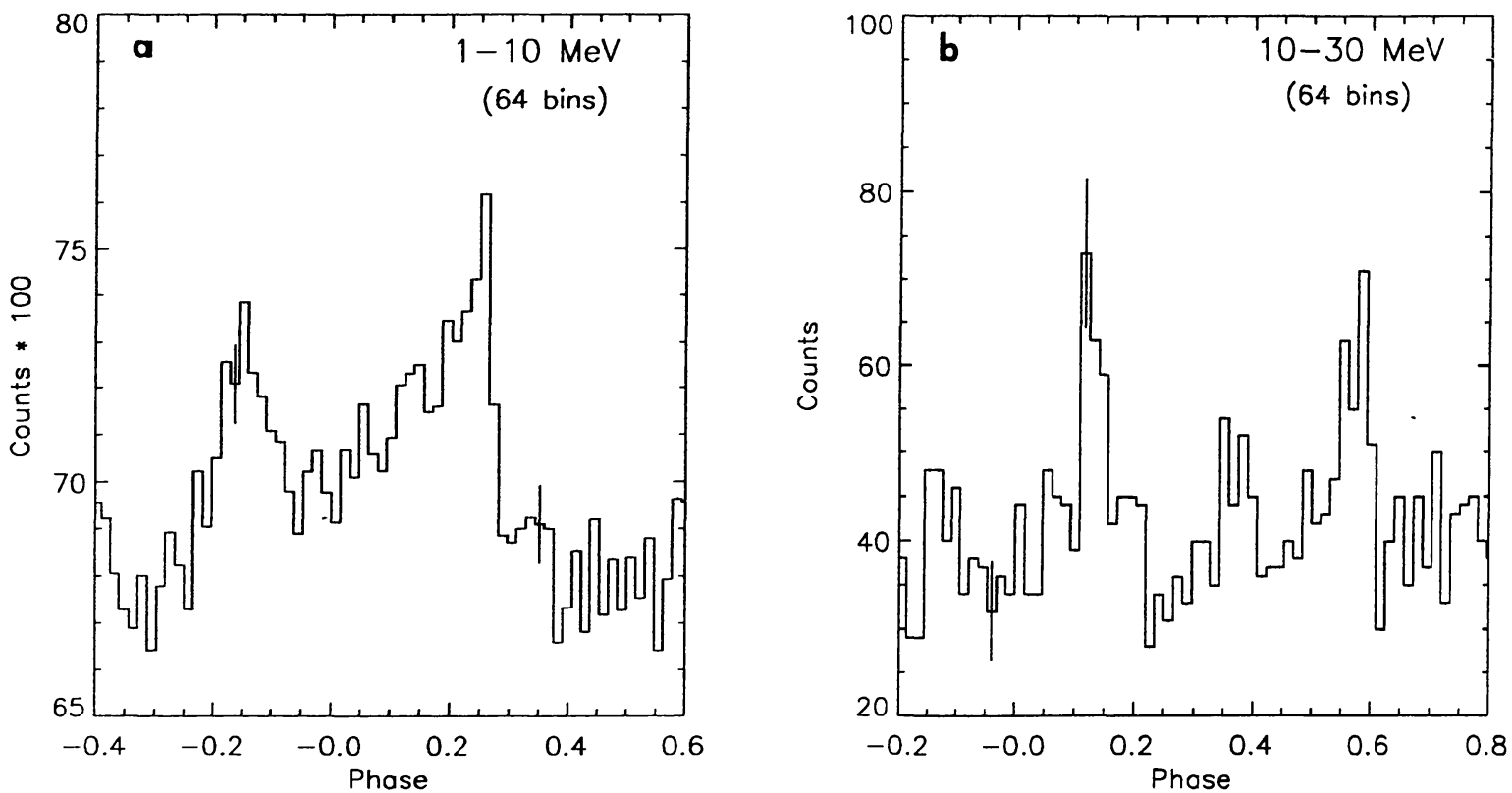

FIG. 1.-COMPTEL light curves: (a) Crab, 1-10 MeV; $(b)$ Vela, 10-30 MeV

the time-average Crab light curve shows more substantial intra-pulse emission than the time-average Vela light curve. Furthermore, the widths of the pulses are significantly different. For Vela, not only is the first peak merely $3.3 \%$ wide (compared with the Crab's $\sim 10 \%$ of the pulse period) but the second peak appears even sharper at $\sim 1 \%$ (Crab $\sim 6 \%$ ). This narrow pulse, becoming visible selecting smaller bins than shown in Figure 1, is the sharpest that we have observed in all gamma-ray light curves.

The spectrum of the Vela pulsar is much harder than that of the Crab. For the latter, pulsed emission can only marginally be detected in the light curve in the 10-30 MeV range, even when combining data of six observations. Contrarily, significant modulation of the Vela light curve can be seen in this range. Hardly any structure is visible for the standard energy intervals $0.7-1 \mathrm{MeV}, 1-3 \mathrm{MeV}$ and 3-10 MeV folding data of four observations (Hermsen et al. 1993). However, selecting events arriving within the pulse interval improves the imaging sensitivity by reducing the background to only that in the pulse interval, and allows to detect weak modulated emission from the pulsar. This approach has been used to prove that the Crab and Vela pulsars are detected over COMPTEL's total energy domain. For example, Figure 2 shows the detection of the Vela pulsar for energies down to $0.75 \mathrm{MeV}$ selecting events from the two main peaks $(\mathrm{P} 1+\mathrm{P} 2)$.

A preliminary COMPTEL Crab spectrum has been presented by Strong et al. (1993), and Ulmer (1993) showed that over seven decades of $\gamma$-ray energy covered by $C G R O$, the Crab spectrum is close to but not fully consistent with a single power law. A similar combined Vela spectrum clearly cannot be represented by a single power law: some bends/breaks are required to represent the data from the EGRET high-energy $\gamma$-rays down to the ROSAT soft X-rays (Fig. 3 ).

The observed temporal variations of the Crab pulses re-

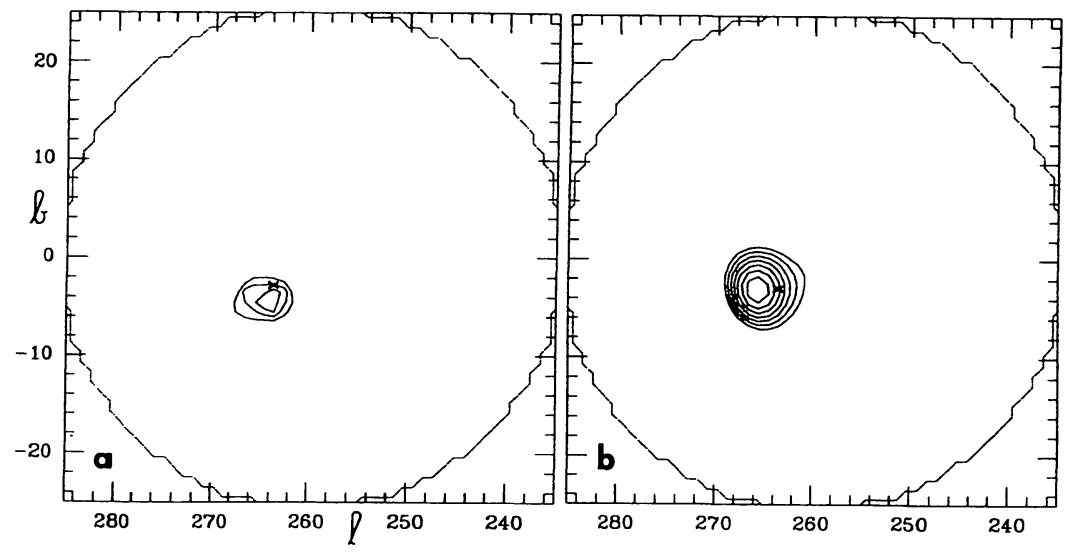

FIG. 2.-Maximum-likelihood images showing Vela (observation 8 only) for events selected in narrow phase intervals around the two peaks ( $\mathrm{P} 1+\mathrm{P} 2)$ in the light curve: (a) 3-10 MeV; (b) 0.75-1 MeV. Contour levels at 15 step 3. 


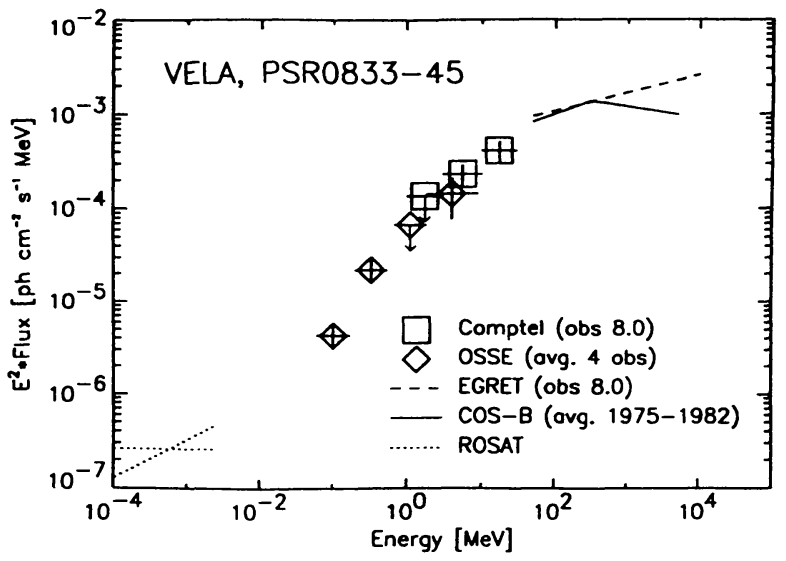

Fig. 3.-Differential Vela spectrum $\left(\times E^{2}\right)$ for the total pulsed emission (references, see Hermsen et al. 1993).

ported by $\operatorname{COS} B$ (Wills et al. 1982) and confirmed by EGRET (Nolan et al. 1993), together with the variations of the Vela pulses, discovered by $\operatorname{COS} B$ (Grenier et al. 1988) and confirmed by EGRET (Kniffen et al. 1993), indicate that the source characteristics should be studied in detail as a function of phase and time. The $\operatorname{COS} B$ findings in the case of Vela show that its pulsed $\gamma$-ray emission is not homogeneous, but is composed of as many as four or five discrete beams of different aperture, spectrum and long-term evolution. This imposes hard constraints on the possible production mechanisms, as discussed in Grenier, Hermsen, \& Henriksen (1993). Nolan et al. (1993) showed that also the Crab light curve $(E \geq 50 \mathrm{MeV})$ contains features similar to Vela's light curve: two different interpulse regions with hard spectra, and a soft trailer after the second peak. Extrapolation to the COMPTEL energies predicts an intense trailer and weak interpulse emission, which is obviously not seen (see Fig. 1). Analysis of phase-resolved COMPTEL spectra is in progress, Figure 4 shows a preliminary spectrum for the first Vela peak (P1) in a single observation in comparison with the time-averaged, broken $\operatorname{COS} B$ spectrum. This COMPTEL P1 spectrum appears to be somewhat harder than that of the total pulsed emission shown in Figure 3.

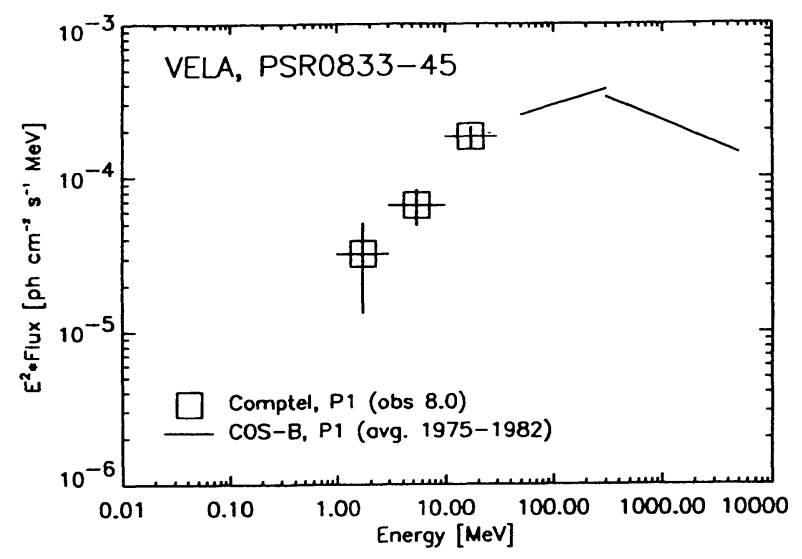

FIG. 4.-Vela spectrum for the first peak (P1) in the light curve

\section{PSR B1509-58, PSR B1706-44 AND PSR B1055-52}

The discovery of PSR B1509-58 in hard X-rays was first reported from Ginga observations by Kawai et al. (1991) for energies below $60 \mathrm{keV}$. CGRO observations with BATSE revealed pulsed emission up to $\sim 700 \mathrm{keV}$ from this object (Wilson et al. 1993) which is the third youngest radio pulsar after the Crab and Vela pulsars, and has the highest dipole magnetic field estimated for any pulsar. The light curve is broad with about a $50 \%$ duty cycle and the spectrum is reported to be harder than that of the Crab. A preliminary analysis of the COMPTEL data on this source (Bennett et al. 1994) rendered a $3 \sigma$ detection of PSR B1509-58 in one observation in the energy range $0.7-1 \mathrm{MeV}$. Figure $5 a$ shows the COMPTEL light curve with a broad peak at the expected phase of 0.97 , as determined by OSSE. The significance of detection is $\sim 3.1 \sigma$ (Gaussian sigmas, testing one harmonic). Figure $5 b$ strengthens the identification: An image of the data selected in a phase interval around the broad peak shows evidence $(\sim 4.3 \sigma)$ for localized emission at the pulsar position, while selection of all events outside the broad peak does not reveal an excess in the skymap. A detailed analysis of all the COMPTEL observations on this source is in progress, and might render constraints on a likely break above $1 \mathrm{MeV}$ in the hard spectrum measured by BATSE (index $-1.8 \pm 0.1$ up to $0.7 \mathrm{MeV}$, Wilson et al. 1993).

The COMPTEL data have also been searched for the new EGRET pulsar PSR B1706-44, so far without success. This source was identified with the hard COS B source 2CG342-02 (Swanenburg et al. 1981) on the basis of the position, and on the hardness of the pulsed $\gamma$-ray emission (Thompson et al. 1992). A similar hard spectrum was measured by EGRET for PSR B1055-52 for energies above $300 \mathrm{MeV}$ (Fierro et al. 1993). It is therefore not surprising that COMPTEL does not see these sources sofar; the hard spectra extrapolate well below the COMPTEL detection threshold.

\section{GEMINGA}

Geminga was in COMPTEL's field-of-view during several observation periods at the start of the all-sky survey. A first search for Geminga in the skymaps was unsuccessful (Strong et al. 1993), as expected from its behavior at higher energies. Namely, the total emission above $50 \mathrm{MeV}$ has proved to be variable between 1975 and 1982 and, in particular, the emission between $50 \mathrm{MeV}$ and $\sim 200 \mathrm{MeV}$ was strongly suppressed most of the time during the $\operatorname{COS} B$ observations (Grenier et al. 1993). The discovery of a $237 \mathrm{~ms}$ period in the EGRET data (Bertsch et al. 1992) stimulated a search for the double-peaked light curve seen by EGRET with a phase separation of 0.5 . Phase-folding of all the available COMPTEL data did not, however, reveal peaks at the EGRET phases, lowering the flux upper limits for the EGRET defined phase intervals (Bennett et al. 1994).

Reanalysis by Grenier et al. (1993a) of the COS B data using the new phase information gave interesting results which are particularly important for the COMPTEL analysis: Four different pulsed components were identified in the Geminga light curve for energies above $50 \mathrm{MeV}$. Two components coincide with the two main peaks ( $\mathrm{P} 1$ and $\mathrm{P} 2$ ), one component falls between the peaks (interpeak 1, I1, the bridge of emission in 

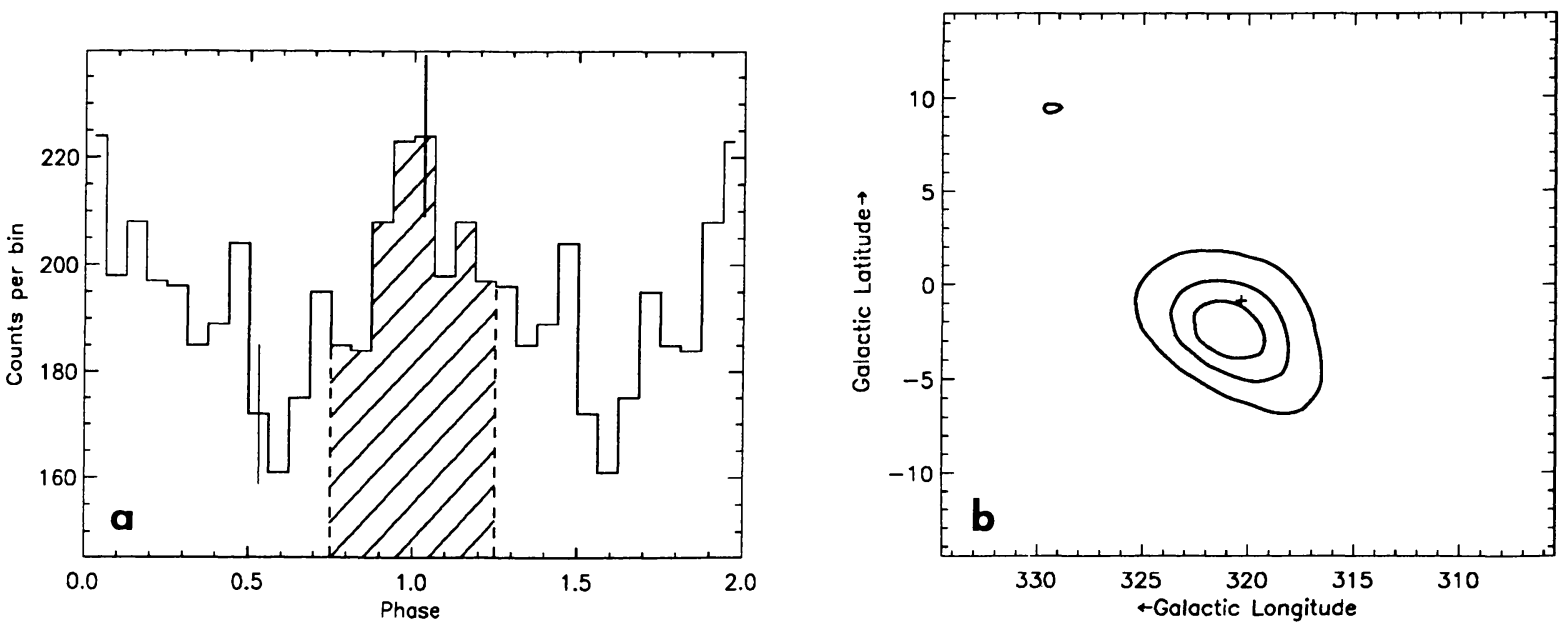

FIG. 5.-Evidence for the COMPTEL detection of PSR B1509-58 in the energy interval 0.75-1.0 MeV: $(a)$ Double light curve with a broad peak at the expected X-ray phase of $0.97 ;(b)$ maximum-likelihood image for events selected in the shaded phase interval in $(a)$. The plus indicates the pulsar position, the contours indicate the 1,2 , and $3 \sigma$ uncertainties on the measured position.
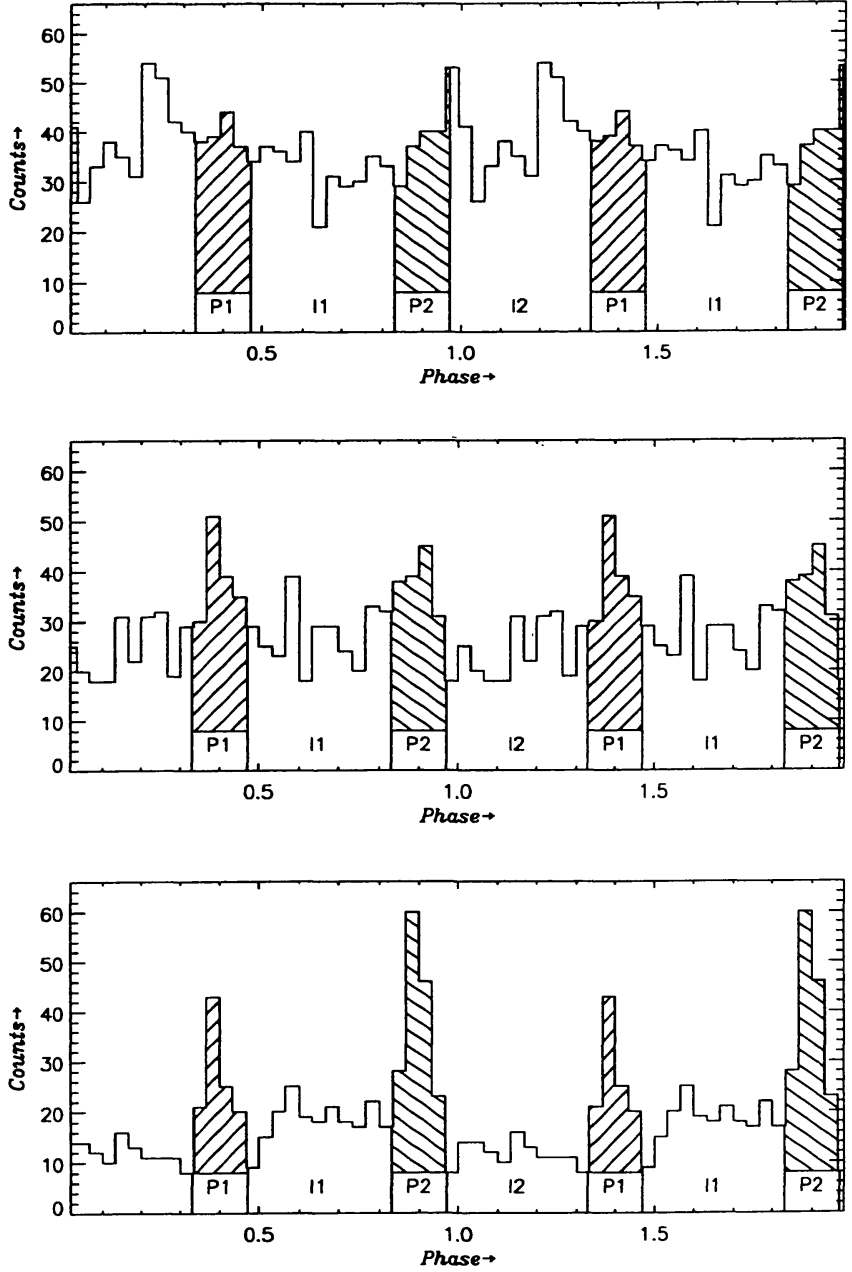

FIG. 6.-Geminga light curves for COMPTEL (Upper figure; validation period, and for energies $10-30 \mathrm{MeV}$ ) and $\operatorname{COS} B$ (Middle figure for 50-200 MeV; lower figure for 200-5000 MeV). The shaded areas mark the phase intervals of the main peaks: $\mathrm{P} 1$ is first peak, P2 is second peak, I1 is interpeak 1 and $\mathrm{I} 2$ is interpeak 2. the EGRET light curve), and the new component Interpeak 2 (I2) has been discovered outside the main peaks. Figures $6 b$ and $6 c$ show the time averaged $\operatorname{COS} B$ light curves for energies above and below $200 \mathrm{MeV}$; the discussed components are indicated. They behave very similarly to four of five components of the Vela light curve. They show significant spectral differences and independent time variability. Figure 7 gives the maximum-likelihood fits to the time-averaged $\operatorname{COS} B$ spectra for each component. Surprisingly, this figure indicates that the best chances for COMPTEL to see Geminga below $30 \mathrm{MeV}$ is in interpeak 2 , outside the two main peaks, where the emission has an average spectral index of $-2.93( \pm 0.25)$. This obviously stimulated a reanalysis of the COMPTEL data.

Geminga was viewed during observations 0 (the validation period), 1 and 2.5. The latter observation was a target-of-opportunity observation of the Sun with the instrument in a special mode. Therefore, a first trial was made using data from observations 0 and 1 for energies 3-10 MeV and $10-30 \mathrm{MeV}$, closest to the $\operatorname{COS} B$ and EGRET energy ranges. Four light curves ( 2 energy ranges, two observations) were produced for standard event selections. Only one light curve, during the validation period, showed an interesting deviation from a flat distribution as measured by the $Z_{3}^{2}$ parameter (Buccheri \& Sacco 1985; see Fig. $6 a$ ). The deviation amounts to $\sim 3 \sigma$ for energies between 10 and $30 \mathrm{MeV}$. The COMPTEL curve has been aligned in phase to the $\operatorname{COS} B$ ones using information on the peak positions in the contemporaneous EGRET light curve. It is evident that no excesses are seen at the phases of the main peaks, but the highest bin is found in interpeak 2 .

Given the softness of the $\operatorname{COS} B$ interpeak-2 component (Fig. 7), and the apparent excess in the COMPTEL light curve, events in this phase interval were selected, and a maximum-likelihood analysis was performed: This showed a weak excess at the position of Geminga. Figure $8 a$ illustrates that the shape of this excess is consistent with that expected for a point source, but the significance in the mapping is only $\geq 2 \sigma$. The flux estimate is given in Figure $8 b$ together with the best powerlaw fits for different $\operatorname{COS} B$ observations (Grenier et al. 1994b). 


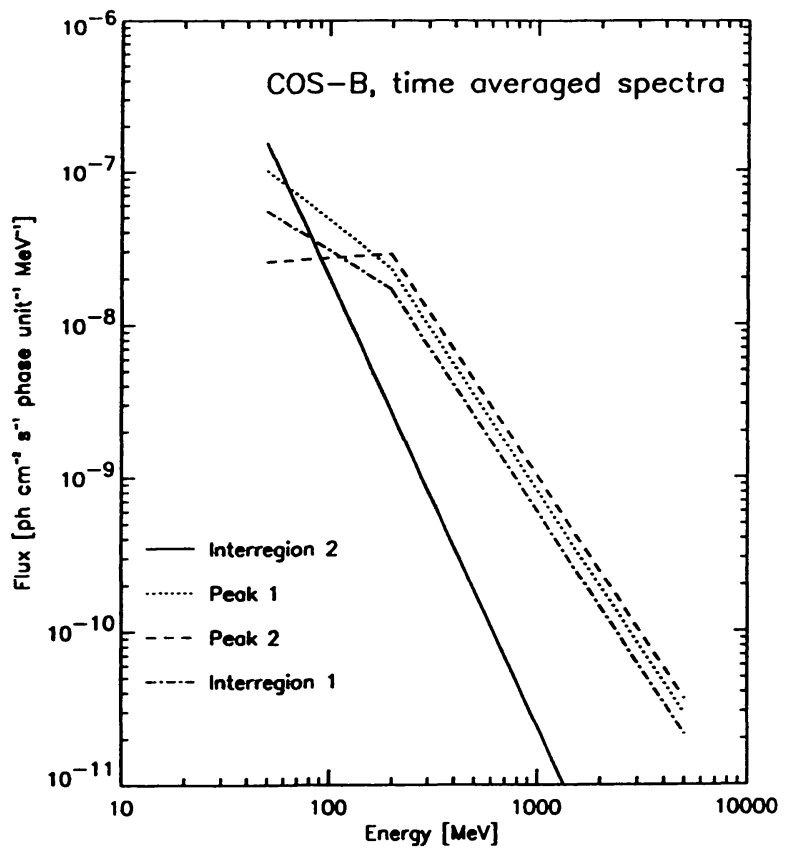

FIG. 7.-Time-averaged spectra fitted to the Geminga emission measured by $\operatorname{COS} B$ in the four phase intervals defined in Fig. 6. For a description of the maximum-likelihood analysis and the uncertainties in the fit parameters see Grenier et al. (1994a).
This preliminary result gives a consistent picture: a likely detection ( $\sim 3 \sigma$ in the timing analysis) of Geminga by COMPTEL in the interpeak 2 of the light curve at a flux level consistent with the soft and variable spectra measured by $C O S$ $B$ for this phase interval. More interesting will be whether the phase resolved EGRET spectra support a weak detection in the COMPTEL data. Further analysis on all available Geminga data is in progress (Kuiper et al. 1994).

\section{SEARCH FOR NEW PULSARS}

We have sorted the $\sim 550$ pulsars in the Princeton Public Data-Base (Johnston et al. 1992) plus the Australian Catalogue (R. N. Manchester 1992, private communication) in terms of $\dot{E} / d^{2}$ as a measure of detectability (for the pulsar catalog see also Taylor, Manchester, \& Lyne 1993). Under the assumption that $I=0.57 \times 10^{45} \mathrm{~g} \mathrm{~cm}^{-2}$ for all pulsars and assuming a $100 \%$ conversion of the power into $\gamma$-rays, we can order the pulsars in terms of the "observed $\gamma$-ray flux" $(F)$ at Earth. From Table 1, which lists the 30 most likely candidates for detection, we can see that objects from the top of the list have been detected already in $\gamma$-rays. Many others have been seen by ROSAT at X-ray energies (Ögelman 1992). Thus far, not all pulsars have been or could be searched for. For a few, however, positive indications were found (see, e.g., Bennett et al. 1993) and refined analysis is in progress.

Since there appears to be a particularly good potential for detection within the top ranks of this list, the reported detection of PSR B1055-52 by EGRET (Fierro et al. 1993) is remarkable for a pulsar listed close to the bottom. This is readily
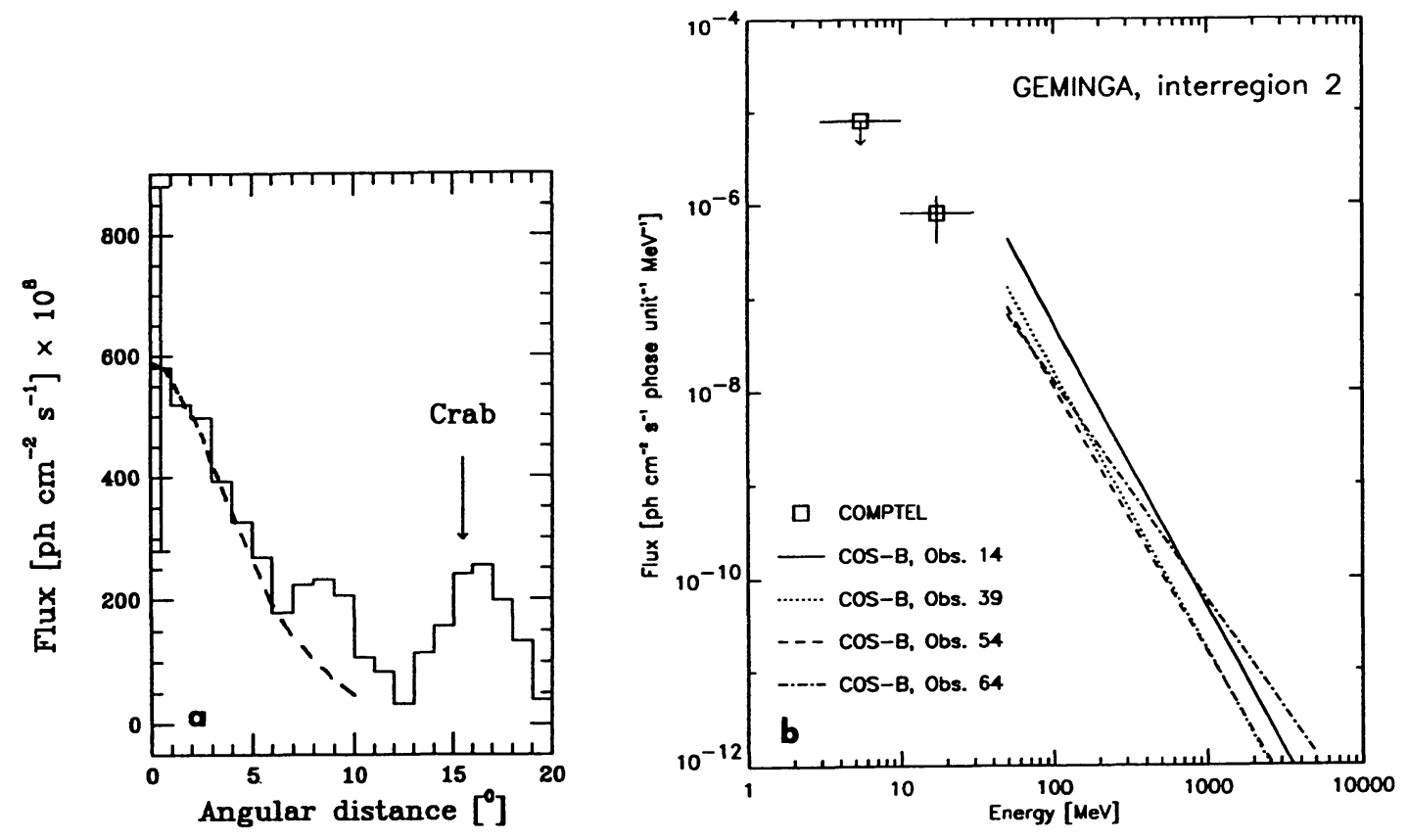

FIG. 8.-COMPTEL results from a spatial and spectral analysis selecting the interpeak 2 component in the Geminga light curve. (a) Radial distribution centered at the position of Geminga, showing the excess in the maximum-likelihood map in comparison with the profile of a point source (the Crab, measured in the same validation period). The error bar represents the statistical error on the measured flux. The Crab is visible at a distance of about $15^{\circ}$ from Geminga (its seemingly lower flux is averaged over the ring with $\sim 15^{\circ}$ radius). (b) Differential spectra of the Interpeak 2 emission of Geminga measured by $\operatorname{COS} B$ in 4 observations of the source (in one observation this component was too weak to be detected by $\operatorname{COS} B$, Grenier et al. 1994b) together with the flux and $2 \sigma$ upper limit measured by COMPTEL in the validation period. 
TABLE 1

COMPTEL PULSAR SEARCh PRIORITy List,

\begin{tabular}{|c|c|c|c|c|c|c|c|c|c|}
\hline & PULSAR & & 1 & b & $\begin{array}{c}P \\
\text { (ms) }\end{array}$ & $\dot{P}$ & $\begin{array}{l}D_{d m} \\
(\mathrm{kpc})\end{array}$ & $\log \left(\dot{E}_{\text {rot }}\right)$ & $\log \left(F_{\max }\right)$ \\
\hline$\gamma$ & $0531+21$ & $\rho$ & 184.56 & -5.78 & 33.342 & $4.212 \times 10^{-13}$ & 2.00 & 38.41 & -6.27 \\
\hline$\gamma$ & $0833-45$ & $\rho$ & 263.55 & -2.79 & 89.286 & $1.243 \times 10^{-13}$ & 0.50 & 36.60 & -6.88 \\
\hline$\gamma$ & GEMINGA & $\rho$ & 195.13 & 4.65 & 237.097 & $1.098 \times 10^{-14}$ & $(0.10)$ & 34.27 & -7.82 \\
\hline \multirow[t]{2}{*}{$\gamma$} & $1706-44$ & $\rho$ & 343.10 & -2.69 & 102.442 & $9.304 \times 10^{-14}$ & 1.46 & 36.29 & -8.12 \\
\hline & $1259-63$ & & 304.18 & -0.99 & 47.762 & $1.850 \times 10^{-14}$ & 2.34 & 36.58 & -8.23 \\
\hline \multirow[t]{21}{*}{$\boldsymbol{\gamma}$} & $1509-58$ & $\rho$ & 320.32 & -1.16 & 150.652 & $1.537 \times 10^{-12}$ & 4.40 & 37.01 & -8.36 \\
\hline & $1929+10$ & $\rho$ & 47.38 & -3.88 & 226.517 & $1.157 \times 10^{-15}$ & 0.08 & 33.35 & -8.48 \\
\hline & $1951+32$ & $\rho$ & 68.77 & 2.82 & 39.530 & $5.849 \times 10^{-15}$ & 2.50 & 36.33 & -8.54 \\
\hline & $1046-58$ & & 287.43 & 0.58 & 123.646 & $9.593 \times 10^{-14}$ & 2.60 & 36.06 & -8.85 \\
\hline & $1823-13$ & $\rho$ & 18.00 & -0.69 & 101.441 & $7.522 \times 10^{-14}$ & 3.98 & 36.21 & -9.07 \\
\hline & $1800-21$ & & 8.40 & 0.15 & 133.588 & $1.341 \times 10^{-13}$ & 3.90 & 36.10 & -9.16 \\
\hline & $1758-24$ & & 5.25 & -0.88 & 124.874 & $1.278 \times 10^{-13}$ & 4.35 & 36.17 & -9.18 \\
\hline & $0740-28$ & & 243.78 & -2.45 & 166.752 & $1.683 \times 10^{-14}$ & 1.10 & 34.91 & -9.25 \\
\hline & $1937+21$ & & 57.51 & -0.29 & 1.558 & $1.051 \times 10^{-19}$ & 3.72 & 35.80 & -9.42 \\
\hline & $1821-24$ & & 7.80 & -5.58 & 3.054 & $1.618 \times 10^{-18}$ & 5.50 & 36.11 & -9.45 \\
\hline & $1727-33$ & & 354.13 & 0.09 & 139.447 & $8.506 \times 10^{-14}$ & 4.13 & 35.85 & -9.46 \\
\hline & $0656+14$ & $\rho$ & 201.11 & 8.26 & 384.875 & $5.503 \times 10^{-14}$ & 0.77 & 34.34 & -9.51 \\
\hline & $1957+20$ & & 59.17 & -4.65 & 1.607 & $1.684 \times 10^{-20}$ & 1.58 & 34.96 & -9.51 \\
\hline & $0540-69$ & $\rho$ & 279.72 & -31.52 & 50.330 & $4.791 \times 10^{-13}$ & 49.40 & 37.93 & -9.54 \\
\hline & $0114+58$ & & 126.28 & -3.46 & 101.437 & $5.845 \times 10^{-15}$ & 2.07 & 35.10 & -9.61 \\
\hline & $1853+01$ & & 34.56 & -0.50 & 267.399 & $2.084 \times 10^{-13}$ & 3.30 & 35.39 & -9.72 \\
\hline & $0950+08$ & $\rho$ & 228.91 & 43.70 & 253.065 & $2.292 \times 10^{-16}$ & 0.13 & 32.50 & -9.80 \\
\hline & $1610-50$ & & 332.21 & 0.18 & 231.591 & $4.923 \times 10^{-13}$ & 6.97 & 35.95 & -9.81 \\
\hline & $1338-62$ & & 308.75 & -0.07 & 193.284 & $2.840 \times 10^{-13}$ & 6.90 & 35.90 & -9.86 \\
\hline & $2127+11 B$ & & 65.01 & -27.31 & 56.133 & $8.800 \times 10^{-15}$ & 10.00 & 36.05 & -10.03 \\
\hline & $1830-08$ & & 23.39 & 0.06 & 85.282 & $9.162 \times 10^{-15}$ & 5.56 & 35.52 & -10.04 \\
\hline \multirow[t]{4}{*}{$\gamma$} & $1055-52$ & $\rho$ & 285.98 & 6.65 & 197.108 & $5.834 \times 10^{-15}$ & 1.38 & 34.24 & -10.12 \\
\hline & $0906-49$ & & 270.27 & -1.02 & 106.757 & $1.514 \times 10^{-14}$ & 5.67 & 35.45 & -10.14 \\
\hline & $1643-43$ & & 341.11 & 0.98 & 231.600 & $1.129 \times 10^{-13}$ & 5.14 & 35.31 & -10.19 \\
\hline & $2334+61$ & & 114.28 & 0.23 & 495.240 & $1.919 \times 10^{-13}$ & 2.40 & 34.55 & -10.28 \\
\hline
\end{tabular}

NOTES. - $\gamma: \gamma$-ray pulsar already detected by $C G R O ; \rho$ : X-ray pulsar already detected by $R O S A T$ (e.g., Ögelman 1992; Finley \& Ögelman 1993). The distance to Geminga is an assumption. Units for $\dot{E}_{\text {rot }}$ ergs s$^{-1}$ and for $F_{\max } \operatorname{ergs~} \mathrm{cm}^{-2} \mathrm{~s}^{-1}$.

visible in Figure $9 a$. The figure shows, for those pulsars seen above $300 \mathrm{MeV}$, the fraction of the total spindown power radiated at $\gamma$-ray energies, the so called $\gamma$-ray efficiency, as a function of pulsar apparent age (this efficiency as a function of period gives a similar distribution ). The $\gamma$-ray luminosities $L_{\gamma}$ were calculated over the energy interval $300-3000 \mathrm{MeV}$, the distances $D$ were taken from Table 1 , and no assumptions were made on the duty cycles (just $4 \pi$ was inserted):

$$
L_{\gamma}=4 \pi D^{2} \int_{300 \mathrm{MeV}}^{3000 \mathrm{MeV}} E \alpha E^{-\beta} d E,
$$

with $\alpha$ and $\beta$ the normalization constant and spectral index, respectively, of the reported power-law spectra. Treating the duty cycle more accurately is difficult, since that requires detailed knowledge of the actual beaming geometries at the source. In fact, the light curve shapes are very different; PSR B1706-44 and PSR B1055-52 show a very broad single peak (Thompson et al. 1992; Fierro et al. 1993), in contrast with the double-peaked light curves of Crab, Vela, and Geminga. The plotted efficiencies can be regarded as upper limits assuming that we do not miss a narrow and intense beam for geometrical reasons. There are obviously additional uncertainties, e.g., in the distance estimate, nevertheless, the $800 \%$ efficiency calculated for PSR B1055-52 (which does not show a narrow beam!), compared to the $12 \%$ value reached for Geminga, and $0.2 \%$ for Crab proves that there is a large spread in the $\gamma$-ray efficiency, as there is for the X-ray efficiency (see Fig. 9b). The 

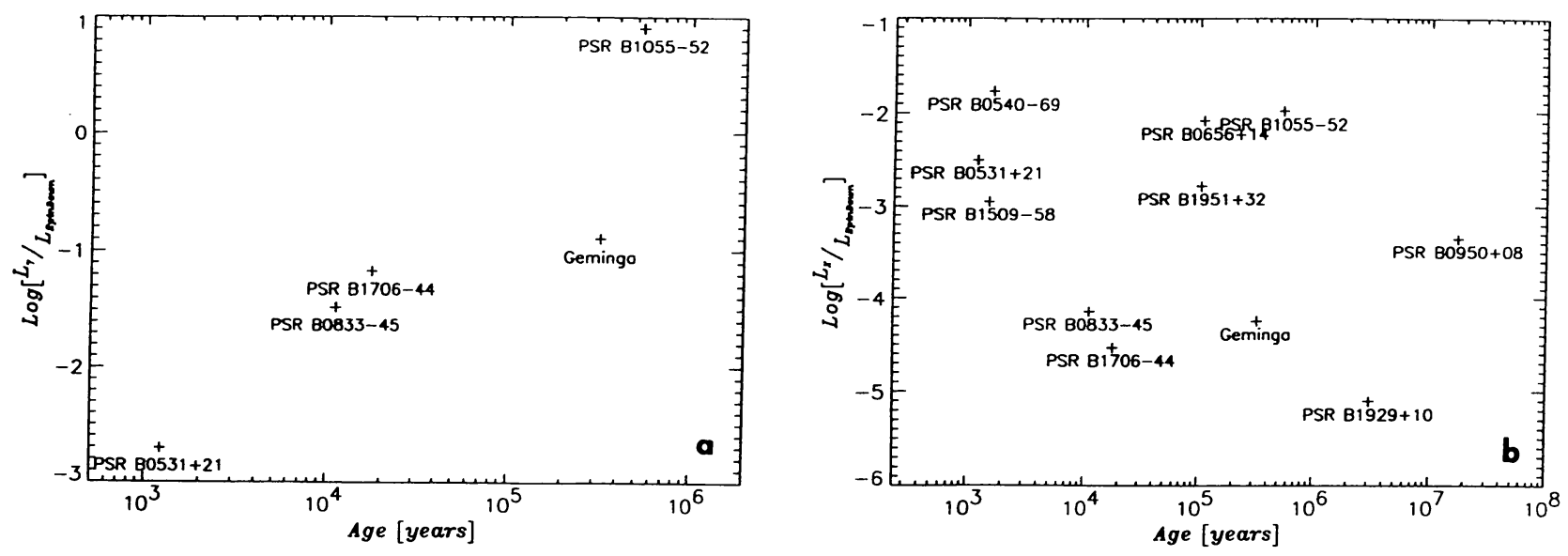

FIG. 9.-Fraction of the total spin-down power radiated in high-energy radiation as a function of pulsar age. $(a)$ For the $\gamma$-ray domain between 300 and $3000 \mathrm{MeV}$. The luminosities for the Crab, Vela, and Geminga are derived from COS B results; for PSR B1706-44 and PSR B1055-52 from EGRET results (for references see text). (b) For the X-ray domain using the results from ROSAT (Ögelman 1992).

apparent spread covers more than three orders of magnitude. PSR B1055-52 is systematically one of the most effiient pulsars in producing high-energy radiation. It is interesting to note the lack of any correlation in Figure $9 b$ for the X-ray emitting pulsars, while a correlation between the $\gamma$-ray efficiency and the age (and period) seems to emerge from the few points in Figure $9 b$. Such a correlation has been discussed for the evolution of gamma-ray pulsars (e.g., Ruderman \& Cheng 1988), however, our sample of gamma-ray pulsars is still very limited and we need more detections to confirm this dependence. Nevertheless, if genuine, the priority list presented in Table 1 significantly underestimates the relative potential for detection of the somewhat older and more slowly rotating pulsars. Therefore, each time an excess is found in the COMPTEL data in the direction of a pulsar, a search for its period in the gamma-ray data will be made, independent of its position in the priority list.

\section{SUMMARY}

The COMPTEL experiment has so far detected three, likely four, of the six $\gamma$-ray pulsars detected by other instruments on $C G R O$. Detailed energy-dependent light curves for the "classical" pulsars Crab and Vela have been derived (Bennett et al. 1993 and Hermsen et al. 1993, respectively). Their spectra will be further studied in detail as a function of phase. Of the new pulsars, detections at the $\sim 4.3$ and $\sim 3 \sigma$ significance level of PSR B1509-58 and Geminga, respectively, are reported. The latter weak detection is consistent with results obtained from a reanalyis of $\operatorname{COS} B$ higher-energy data. Grenier et al. (1988) and Grenier et al. (1994a) concluded that the Vela and Geminga $\gamma$-ray emissions consist of five and four, respectively, discrete beams of different aperture and spectra ( see for a similar result for Crab Nolan et al. 1993). The first phase-resolved spectra derived from the COMPTEL data for Vela connect smoothly to the corresponding time-averaged $\operatorname{COS} B$ spectra. The COMPTEL flux measured for Geminga in the so called interpeak 2 is also consistent with the extrapolation of its timeaveraged spectrum measured by $C O S B$. The implied breaks in the time variable spectra of Vela and Geminga, going from the $\operatorname{COS} B$ /EGRET range to the COMPTEL MeV energies, may find its roots in the properties of curvature radiation or very small pitch-angle synchroton radiation, as discussed by Grenier et al. (1993). So far, the diversity in light-curve shapes, spectra, time variabilities and the significant differences in the efficiency to produce high-energy radiation do not present a coherent picture. These results bring forward the importance of the simultaneous measurements by the instruments aboard CGRO. So far, most of their results have not yet been published consistently and in full detail. Interpretation of the combined detailed results should help to unravel the underlying pulsar emission mechanisms.

\section{REFERENCES}

Akimov, V., et al. 1993, in Proc. COSPAR Gamma-ray Astronomy Symp., Advances in Space Research, Vol. 13, No. 12, ed. G. G. C. Palumbo, G. F. Bignami, (Oxford: Pergamon)

Bennett, K., et al. 1993, A\&AS, 97, 317 . 1994, in Proc. IAU Symp. 142, Particle Acceleration Phenomena

in Astrophysical Plasmas, ApJS, 90, 823

Bertsch, D. L., et al. 1992, Nature, 357, 306

Bignami, G. F., \& Caraveo, P. A. 1992, Nature, 357, 287

Buccheri, R., \& Sacco, B. 1985, in Data Analysis in Astronomy (New

York: Plenum Press)

Cheng, K. S., Ho, C., \& Ruderman, M. 1986a, ApJ, 300, 500

. 1986b, ApJ, 300, 522
Clear, J., et al. 1987, A\&A, 174, 85

Daugherty, J. K., \& Harding, A. K. 1982, ApJ, 252, 337

Fierro, J. M., et al. 1993, ApJ, 413, L27

Finley, J. P., \& Ögelman, H. 1993, IAU Circ., 5787

Grenier, I. A., Hermsen, W., \& Clear, J. 1988, A\&A, 204, 117

Grenier, I. A., Hermsen, W., \& Henriksen, R. N. 1993, A\&A, 269, 209

Grenier, I. A., et al. 1994a, in IAU Symp. 142, Particle Acceleration Phenomena in Astrophysical Plasmas, ApJS, 90, 813 1994b, A\&A, in preparation

Grenier, I. A., Bennett, K., Buccheri, R., Gros, M., Henriksen, R. N., Hermsen, W., Kanbach, G., \& Sacco, B. 1994c, unpublished

Halpern, J. P., \& Holt, S. S. 1992, Nature, 357, 222 
Hermsen, W, et al. 1992, IAU Circ., 5541

1993, in AIP Conf. Proc. 280, Compton Gamma-Ray Observatory, ed. M. Friedlander, N. Gehrels, \& D. J. Macomb (New York: AIP), 204 Johnston, S., et al. 1992, CGRO/Radio Timing Database, Princeton University

Johnston, S., Manchester, R. N., Lyne, A. G., Bailes, M., Kaspi, V. M., $\underset{1 \rightarrow 1}{ }$ Guojun, Q., \& D’Amico, N. 1992, ApJ, 387, L37

Kawai, N., et al. 1991, ApJ, 383, 645

Kniffen, D. K., et al., 1993, in AIP Conf. Proc. 280, Compton Gamma-Ray Observatory, ed. M. Friedlander, N. Gehrels, \& D. J. Macomb (New York: AIP), 177

Kuiper, L., et al. (COMPTEL Coll.), 1994, A\&A, in preparation

Nolan, P. L., et al. 1993, ApJ, 409, 697
Ögelman, H., 1992, private communication Ruderman, M., \& Cheng, K. S. 1988, ApJ, 335, 306

Strong, A. W., et al. 1993, A\&AS, 97, 133

Swanenburg, B. N., et al. 1981, ApJ, 243, L69

Taylor, J. H., Manchester, R. N., \& Lyne, A. G. 1993, ApJS, 88, 529

Thompson, D. J., et al. 1992, Nature, 296, 723

Ulmer, M. 1993, in IAU Symp. 142, Particle Acceleration Phenomena in Astrophysical Plasmas, ApJS, 90, 789

Wills, R. D., et al. 1982, Nature, 296, 723

Wilson, R., et al. 1991, BAAS, 23:4, 57.07

. 1993, in Proc. of Los Alamos Workshop of Physics of Isolated Pulsars, ed. K. A. van Riper, R. Epstein, \& C. Ho (Cambridge: Cambridge Univ. Press), 257 